\title{
Treatment of Electronic Energy Level Transition and Ionization Following the Particle-Based Chemistry Model
}

\author{
Derek S. Liechty ${ }^{* \dagger}$ \\ NASA Langley Research Center, Hampton, VA 23681 \\ Mark Lewis \\ University of Maryland, College Park, MD 20742
}

\begin{abstract}
A new method of treating electronic energy level transitions as well as linking ionization to electronic energy levels is proposed following the particle-based chemistry model of Bird. Although the use of electronic energy levels and ionization reactions in DSMC are not new ideas, the current method of selecting what level to transition to, how to reproduce transition rates, and the linking of the electronic energy levels to ionization are, to the author's knowledge, novel concepts. The resulting equilibrium temperatures are shown to remain constant, and the electronic energy level distributions are shown to reproduce the Boltzmann distribution. The electronic energy level transition rates and ionization rates due to electron impacts are shown to reproduce theoretical and measured rates. The rates due to heavy particle impacts, while not as favorable as the electron impact rates, compare favorably to values from the literature. Thus, these new extensions to the particle-based chemistry model of Bird provide an accurate method for predicting electronic energy level transition and ionization rates in gases.
\end{abstract}

\section{Nomenclature}

$d \quad=$ diameter of particle $(\mathrm{m})$

$d n \quad=$ number of transitions in sample

$d t \quad=$ sample time $(\mathrm{s})$

$f \quad=$ distribution function

$g \quad=$ energy level degeneracy

$i, j, J \quad=$ energy levels

$k=$ Boltzmann's Constant $\left(k=1.3806503 \mathrm{e}-23 \mathrm{~m}^{2} \mathrm{~kg} \mathrm{~s}^{-2} \mathrm{~K}^{-1}\right)$

$k_{f}=$ forward reaction rate $\left(\mathrm{m}^{3} / \mathrm{molecule} / \mathrm{s}\right)$

$l=$ orbital quantum number

$L=$ total orbital angular momentum quantum number

$m \quad=\operatorname{mass}(\mathrm{kg})$

$N \quad=$ number of molecules

$n=$ number density $\left(1 / \mathrm{m}^{3}\right)$ and principal quantum number

$P \quad=$ probability

$S \quad=$ total spin quantum number

$T \quad=$ temperature $(\mathrm{K})$

$v_{r} \quad=$ relative velocity in a collision $(\mathrm{m} / \mathrm{s})$

$x=$ number of active electrons for an atomic level

$\delta \quad=$ Dirac delta

$\varepsilon \quad=\operatorname{energy}(\mathrm{J})$

$\varepsilon_{t r} \quad=$ translational energy $\left(\varepsilon_{t r}=0.5 \mu v_{r}^{2}\right)$

$\Gamma \quad=$ gamma function

$\mu \quad=$ reduced mass in a collision $\left(\mu=m_{1} m_{2} /\left(m_{1}+m_{2}\right)\right)$

$\omega \quad=$ exponent in variable hard sphere model

\footnotetext{
*Aerospace Engineer, Aerothermodynamics Branch, MS 408A, AIAA Member.

$\dagger$ Graduate Student, University of Maryland, Aerospace Engineering

$\$$ Professor, Aerospace Engineering, Room 3179 Martin Hall, AIAA Fellow, AIAA President-Elect
} 




\section{Introduction}

As we consider missions, both manned and unmanned, where the (re)entry velocities result in shock-layer temperatures on the order of tens-of-thousands of degrees Kelvin, the importance of electronic energy levels and ionization reactions become more pronounced. Although the treatment of electronic energy levels and ionization reactions using the direct simulation Monte Carlo (DSMC) method is not a new development ${ }^{1-3}$, most of these methods are based on measured, equilibrium rates, which are always questionable at high temperature, especially when applied to non-equilibrium problems. The primary reason for the inadequate state of chemical reaction modeling is the difficulty in accurately measuring the internal energy state specific reaction rates to validate theoretical models in the temperature range of interest. Therefore, most reaction rates are based on low-temperature equilibrium measurements and are fit to the most reliable measured data sets. In some cases, the uncertainty associated with the spread of these measurements exceeds one order of magnitude 4 .

Recently, an approach for determining chemical reaction rates from microscopic molecular data, referred to as the particle-based chemistry model (PBM), has been developed ${ }^{5,6}$ that does not use any macroscopic rate information. It is the purpose of this paper to introduce a new method of treating electronic energy level transitions following the PBM methodologies. One distinct feature of the PBM is the direct linking of the vibrational energy level of a molecule to the dissociation of that molecule. This idea is extended in this paper to the direct linking of the electronic energy level of an atom/molecule to ionization.

\section{Detailed Electronic Energy Level Model for DSMC}

In order to implement a model for the distribution of electronic energy in the DSMC technique, there are three procedures that must be defined. First, when a particle is first introduced into a simulation, it is necessary to obtain a new electronic energy from a given distribution through statistical sampling. This is also required when a particle changes its electronic energy after a collision with a surface. Under equilibrium conditions, the distribution has the well known Boltzmann form. Second, it is necessary to statistically sample a new electronic energy following a collision which involves electronic energy transfer. Finally, a method to reproduce the electronic energy transition rate must be defined. In the following subsections, each of these tasks are considered.

\section{A. Equilibrium Sampling}

Each electronic energy level $j$ has a distinct energy, $\varepsilon_{j}$, and degeneracy, $g_{j}$. The Boltzmann distribution for the electronic energy levels at a given temperature $T$ gives the following result for the fraction of particles in level $j$ :

$$
f_{e l}(j)=\frac{N_{j}}{N}=\frac{g_{j} \exp \left(-\varepsilon_{j} / k T\right)}{\sum_{i=0}^{i_{\max }} g_{i} \exp \left(-\varepsilon_{i} / k T\right)}
$$

This distribution is used when creating a new particle in the DSMC simulation at a boundary specified at the temperature $T$. However, it is not possible to sample an electronic energy level $j$ directly from the distribution. 
Therefore, an acceptance-rejection procedure is used. This is performed by selecting values for $j$ from the following distribution:

$$
f_{e l}^{\prime}(j)=\frac{g_{j} \exp \left(-\varepsilon_{j} / k T\right)}{g_{J} \exp \left(-\varepsilon_{J} / k T\right)}
$$

where $J$ is the value of $j$ for which Eq. (1) is a maximum. Unfortunately, since the degeneracy of each level is a variable specific to each species, there is no way to know a priori what level is going to give the maximum, so the maximum level must be searched for at each implementation or saved for a constant boundary temperature. The sampling of a new electronic energy level then proceeds as follows:

1. select at random an electronic energy level evenly distributed between 0 and $J_{\max }$, where $J_{\max }$ is the maximum possible energy level. A random number, RAND ${ }_{1}$, evenly distributed between 0 and 1 is needed such that:

$$
j=\operatorname{int}\left[\left(1+J_{\max }\right) * R A N D_{1}\right]
$$

2. determine the value $J$ for which Eq. (1) is a maximum;

3. accept the value of $j$ if $f_{e l}^{\prime}(j)>\mathrm{RAND}_{2}$;

4. if the value of $j$ is not accepted, then return to step 1 .

\section{B. Post-collision sampling}

A phenomenological approach is usually adopted in the DSMC method when a collision occurs that involves energy transfer. The Borgnakke-Larsen $\operatorname{method}^{7}$ samples a post-collision state from a combined distribution of the translational and electronic collision energies of the colliding particles. Based on the approach of Bergemann and $\mathrm{Boyd}^{8}$ and $\mathrm{Boyd}^{9}$, the Dirac delta is used to write the distribution of energies in Eq. (1) in the following continuous form:

$$
f_{e l}\left(\varepsilon_{e l}, j\right)=\frac{g_{j} \exp \left(-\varepsilon_{j} / k T\right)}{\sum_{i=0}^{i_{\max }} g_{i} \exp \left(-\varepsilon_{i} / k T\right)} \delta\left(\varepsilon_{e l} / k T-\varepsilon_{e l}^{j} / k T\right)
$$

Consideration must now be given to the distribution of translational energy of the colliding particles. This distribution is naturally affected by the intermolecular model used since this determines the collision probability. For the present study, the variable hard sphere (VHS) collision model of Bird ${ }^{10}$ is used. However, it is a simple matter to develop the formulation for an alternative collision model such as the variable soft sphere of Koura ${ }^{11}$. For the VHS model, the distribution of the translational collision energies is:

$$
f_{t r}\left(\varepsilon_{t r}\right)=\frac{1}{\Gamma(5 / 2-\omega)}\left(\varepsilon_{t r} / k T\right)^{3 / 2-\omega} \exp \left(-\varepsilon_{t r} / k T\right)
$$

Using Eqs. (4) and (5), the combined distribution for sampling a post-collision electronic energy level $j$ ' from the total collision energy $\varepsilon_{c o l l}=\varepsilon_{t r}+\varepsilon_{e l}=\varepsilon^{\prime}{ }_{t r}+\varepsilon^{\prime}{ }_{e l}$ is:

$$
f\left(j^{\prime} ; \varepsilon_{\text {coll }}\right)=\frac{1}{\Gamma(5 / 2-\omega)} \frac{g_{j}}{\sum_{i=0}^{i_{\max }} g_{i} \exp \left(-\varepsilon_{i} / k T\right)}\left[\left(\varepsilon_{\text {coll }}-\varepsilon_{\text {el }}^{\prime}\right) / k T\right]^{3 / 2-\omega} \exp \left(-\varepsilon_{\text {coll }} / k T\right)
$$


In applying the general Borgnakke-Larsen scheme, it is assumed that local thermodynamic equilibrium prevails. Therefore, the temperature $T$ in Eq. (6) is constant. Also, the total collision energy is constant, so it is only necessary to perform sampling of the post-collision state from the following distribution form:

$$
g\left(j^{\prime} ; \varepsilon_{\text {coll }}\right) \propto g_{j^{\prime}}\left(\varepsilon_{\text {coll }}-\varepsilon_{e l}^{j^{\prime}}\right)^{3 / 2-\omega}
$$

Again, an acceptance-rejection procedure is used. The normalized distribution that is required is obtained by finding the value of $j$ ' for which Eq. (7) is maximum, $J$ ', which is different for each value of $\varepsilon_{\text {coll }}$. Therefore, the following distribution is obtained:

$$
g^{\prime}\left(j^{\prime} ; \varepsilon_{\text {coll }}\right)=\frac{g_{j^{\prime}}\left(\varepsilon_{\text {coll }}-\varepsilon_{e l}^{j^{\prime}}\right)^{3 / 2-\omega}}{g_{J^{\prime}}\left(\varepsilon_{\text {coll }}-\varepsilon_{e l}^{J^{\prime}}\right)^{3 / 2-\omega}}
$$

In the DSMC code, these procedures are implemented in the following way:

1. Given a pair of particles with total collision energy $\varepsilon_{\text {coll }}$ which undergoes electronic energy exchange, determine $J^{\prime}$;

2. determine the maximum allowable electronic energy level obtainable from $\varepsilon_{c o l l}, J$;

3. take $J^{*}$ to be the smaller of $J^{\prime}$ and $J^{\prime \prime}$;

4. as described in the previous section on equilibrium sampling, perform an acceptance-rejection procedure to sample $j$ ' from:

$$
g^{\prime}\left(j^{\prime} ; \varepsilon_{\text {coll }}\right)=\frac{g_{j^{\prime}}\left(\varepsilon_{c o l l}-\varepsilon_{e l}^{j^{\prime}}\right)^{3 / 2-\omega}}{g_{J^{*}}\left(\varepsilon_{\text {coll }}-\varepsilon_{e l}^{J^{*}}\right)^{3 / 2-\omega}}
$$

\section{Electronic Energy Level Transitions}

A transition from electronic energy level $i$ to level $j$ for atomic nitrogen, $N$, can be written in the form of a chemical reaction as:

$$
N^{i}+M \rightarrow N^{j}+M
$$

Following the PBM, the simplest model is to assume that the transition occurs if the electronic energy level of $N$ after a trial Borgnakke-Larsen redistribution of the collision energy is $j^{*}=j$. Since we are treating the transition as a chemical reaction, there are $J_{\max }$ transitions to consider where $J_{\max }$ is the maximum energy level energetically possible. There is a possibility that there will be multiple transitions where $j^{*}=j$, each of which, according to the PBM, are equally probable. In the DSMC code, the decision to transition from level $i$ to $j$ is implemented in the following way:

1. check each transition $i$ to $j$ from $j=0$ to $J_{\max }$, performing a trial Borgnakke-Larsen redistribution for each, keeping a list of the possible reactions $\left(j^{*}=j\right)$.

2. if there are any possible transitions from step (1), choose a random transition from the list and perform the transition.

\section{Linking Ionization to Electronic Energy Levels in DSMC}

Bird $^{5}$ proposed a set of molecular-level chemistry models based solely on fundamental properties of the two colliding molecules: their total collision energy, quantized vibrational energy levels, and molecular dissociation energies. These models link chemical-reaction cross sections to the energy exchange process and the probability of transition between vibrational energy states. The Borgnakke-Larsen procedures and the principle of microscopic reversibility are then used to derive simple models for recombination reactions and for the reverse (exothermic) reactions. These models do not require any macroscopic data, and they function by seeking to balance the fluxes into and out of each state, thus satisfying microscopic reversibility. 
Table 1. Gas properties.

\begin{tabular}{c|c|c|c|c|c|c|c|c|}
\hline Test Gas & $\boldsymbol{d}_{\boldsymbol{r e f}}(\mathbf{m})$ & $\boldsymbol{m} \mathbf{( k g )}$ & $\boldsymbol{T}_{\boldsymbol{r e f}}(\mathbf{K})$ & $\boldsymbol{\omega}$ & $\boldsymbol{\Theta}_{\text {vib }}(\mathbf{K})$ & $\boldsymbol{Z}_{\boldsymbol{r e f}}$ & $\boldsymbol{\Theta}_{\text {diss }}(\mathbf{K})$ & $\boldsymbol{\varepsilon}_{\text {ion }}(\mathbf{J})$ \\
\hline $\mathbf{N 2}$ & $3.580 \mathrm{E}-10$ & $4.650 \mathrm{E}-26$ & 1000 & 0.68 & 3371.0 & 52500 & 113500 & $2.496192 \mathrm{E}-18$ \\
\hline $\mathbf{N}$ & $3.110 \mathrm{E}-10$ & $2.325 \mathrm{E}-26$ & 1000 & 0.65 & - & - & - & $2.327964 \mathrm{E}-18$ \\
\hline $\mathbf{e}$ & $5.640 \mathrm{E}-15$ & $9.109 \mathrm{E}-31$ & 1000 & 0.60 & - & - & - & - \\
\hline
\end{tabular}

In this section, these ideas are extended to include the quantized electronic energy levels and molecular/atomic ionization energies. For dissociation to occur, if the collision energy:

$$
\varepsilon_{\text {coll }}=\varepsilon_{\text {tr,pair }}+\varepsilon_{\text {vib,particle }}
$$

exceeds the dissociation energy, the molecule in question dissociates. If this is extended to electronic energy levels and ionization, if the collision energy now defined as:

$$
\varepsilon_{\text {coll }}=\varepsilon_{\text {tr,pair }}+\varepsilon_{\text {el,particle }}
$$

exceeds the ionization energy, the particle in question loses an electron, becoming an ion.

\section{Results}

In this section, the new electronic energy exchange, electronic energy level transition, and ionization reaction models are applied in a two-dimensional DSMC code where the results are accumulated over all cells to approximate a zero-dimensional simulation. The test gas is comprised of molecular and atomic nitrogen, $N_{2}$ and $N$, and electrons, $e$, where the relevant simulation parameters are listed in Table 1. A list of electronic energy levels for $\mathrm{N}_{2}$ and $\mathrm{N}$ are presented in Tables A.1 and A.2 in the Appendix, respectively. The simulations were run at varying temperatures between $10,000 \mathrm{~K}$ and $60,000 \mathrm{~K}$ at a number density of $1 \mathrm{e} 23 / \mathrm{m}^{3}$ in an adiabatic box $0.002 \mathrm{~m}$ on a side with 300,000 molecules. During the simulations, relaxation of the particles was allowed to proceed as usual, but when a reaction was determined to take place, the number of reactions was advanced by one but the simulators were left unchanged, so no energy was added to or taken away from the flow. In the figures included in this section, the solid lines are values from quoted rates from the literature, and the symbols are the values that were sampled from DSMC.

\section{A. Electronic Energy Level Transitions and Comparison to Equilibrium}

In order for these procedures to be acceptable, they need to reproduce equilibrium conditions after the simulation has been allowed to run for a sufficient amount of time. This set of simulations were allowed to proceed for approximately 24 hours, resulting in at least 300 millions collisions. The first requirement is for the procedures to remain at the input equilibrium temperature. The input equilibrium temperature is compared to the sampled translational temperature in Fig. 1 and can be seen to remain constant. The resultant population distribution over the first twenty energy levels for a range of temperatures is compared to the Bolzmann distribution in Fig. 2. Again, the simulation represents the equilibrium state very well.

Sampled transition rates will now be compared to those found in literature. We must first define how to measure the sampled transition rates in DSMC. The transition rate for the reaction shown in Eq. (10) is calculated as:

$$
k_{f}=\frac{\frac{d n_{N^{i}}}{d t}}{n_{N^{i}} n_{N}}
$$



Figure 1. Sampled translational temperature over a range of input equilibrium temperatures. 

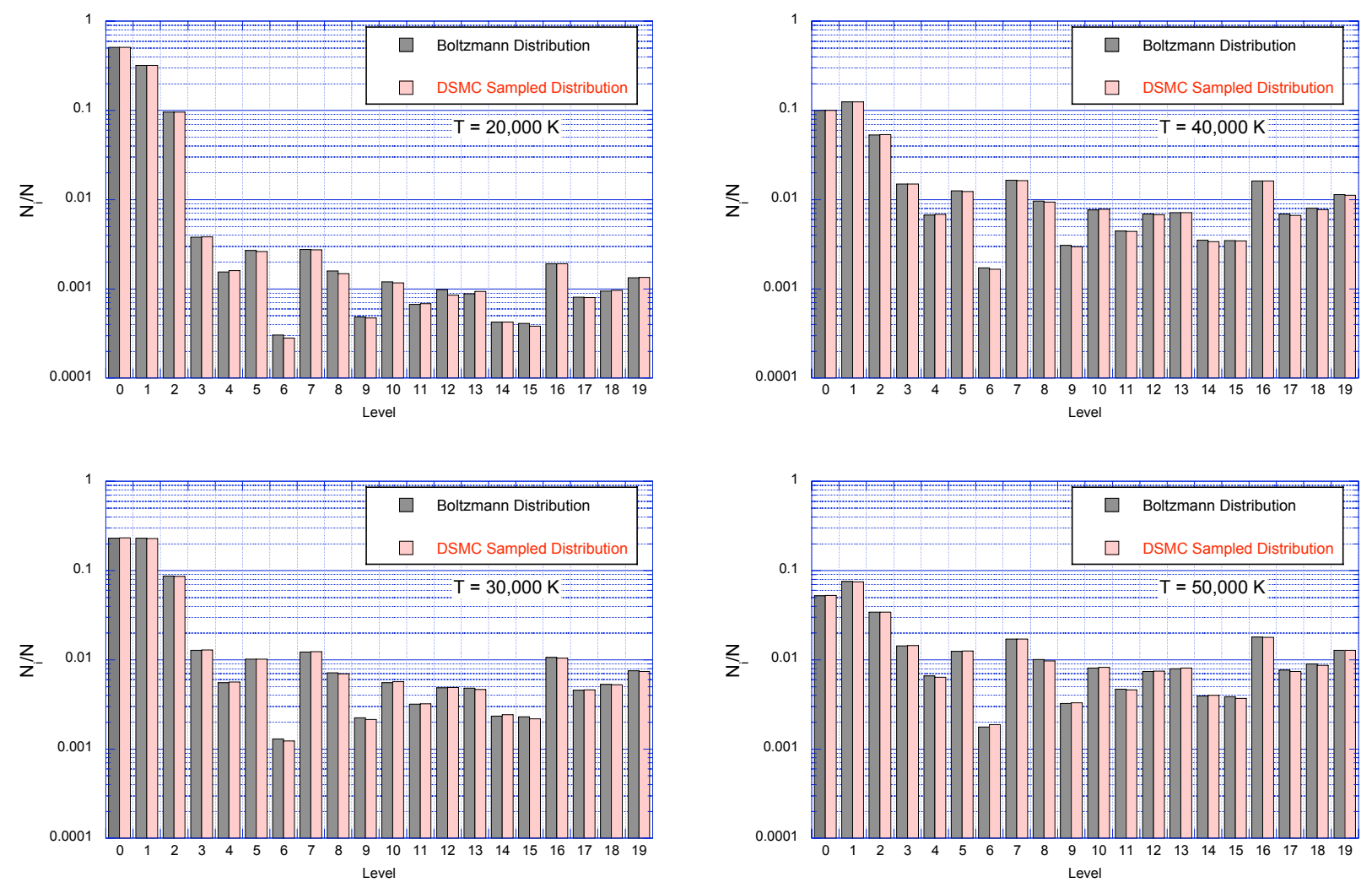

Figure 2. Sampled electronic energy level distributions for the first 20 levels of atomic nitrogen over a range of equilibrium temperatures.

where the numerator is the change in the number density of level $i$ due to the reaction in the sampled time.

1. Electron impact rates

We will begin by examining electronic energy level transitions caused by electron impacts. Sampled transition rates are compared to those by Chernyi ${ }^{12}$ in Fig. 3 for molecular nitrogen. The sampled rates very nearly match the values quoted in the literature. Sampled electron impact electronic energy level transition rates for atomic nitrogen are compared in Fig. 4 with values from Frost ${ }^{13}$ with similar results.

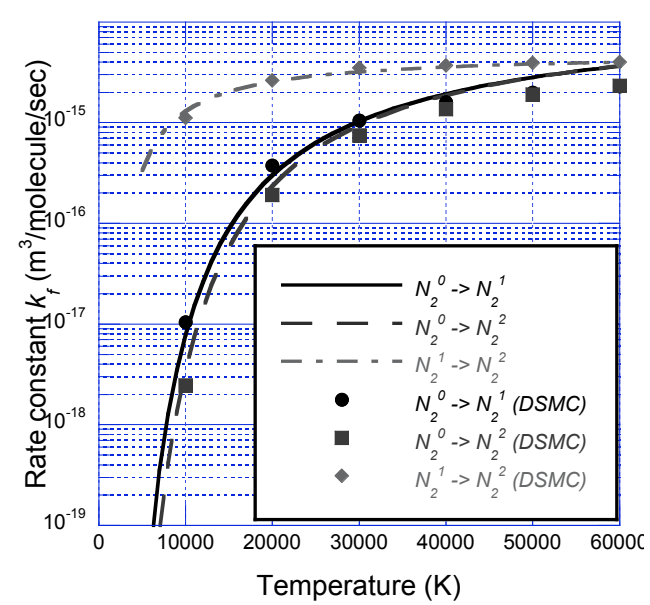

Figure 3. Electron impact electronic energy transition rates for $N_{2}$.

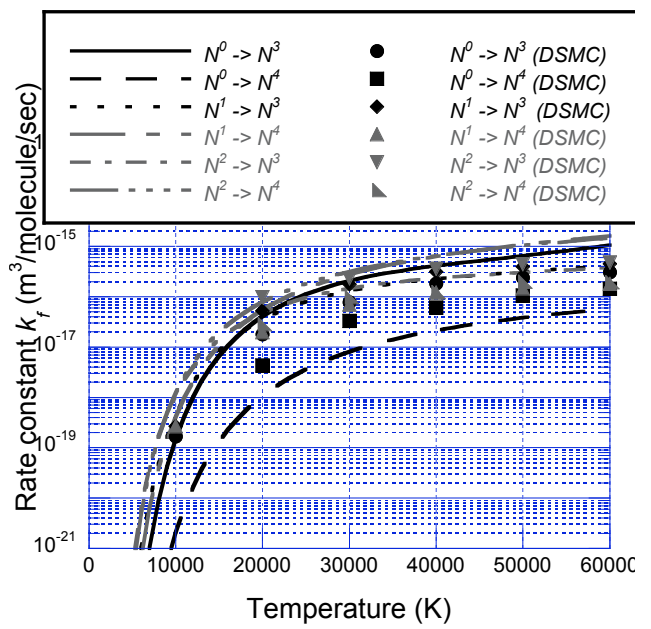

Figure 4. Electron impact electronic energy transition rates for $N$. 


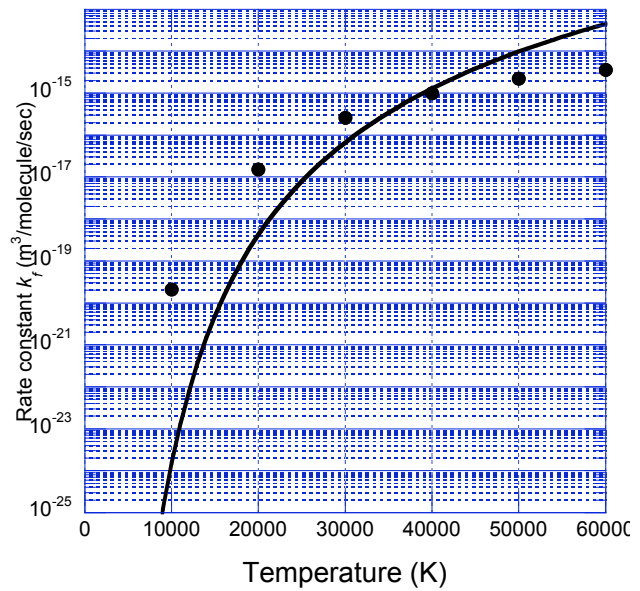

Figure 5. Electron impact ionization rates for $N_{2}$.



Figure 6. Electron impact ionization rates for $N$.

Next, electron impact ionization rates will be compared for both atomic and molecular nitrogen. Figure 5 presents a comparison between the sampled rates and those from Losev ${ }^{14}$ for molecular nitrogen while Figure 6 compares the rates between DSMC and a variety of sources, ${ }^{4}$-19 for electron impact ionization rates for atomic nitrogen. As with the electronic energy level transition rates for electron impact, the ionization rates compare well with literature.

\section{Heavy particle impact rates}

Using the current model for electronic energy level transitions and ionization, the comparison between rates measured using DSMC and those from the literature for heavy particle impacts are not as similar as those for electron impacts. Sampled heavy particle impact endothermic electronic energy level transition rates for molecular nitrogen are compared to those of Park ${ }^{20}$ in Fig. 7. The measured trends match those from the literature for individual level transitions, but the magnitudes are different, especially for the level 0 to 1 transition for a $N_{2}+N_{2}$ collision. The other transition rates are approximately within an order of magnitude of the quoted rates, but the comparison is not as good as the electron impact transition rates. Sampled heavy particle impact exothermic electronic energy level transition rates for $N_{2}$ are compared to those of Guerra ${ }^{21}$ in Fig. 8. The quenching transitions are much closer to the measured rates as compared to the excitation transitions. This is most likely due to the fact that it is the quenching rates that are measured in the laboratory. The excitation rates are then calculated assuming equilibrium. It is possible that the transitions in the laboratory are not at equilibrium, therefore some of the rates are farther off than others.

When examining atomic nitrogen heavy particle impact electronic energy level transition rates, only quenching transition rates were found for comparison to the sampled rates. The sampled rates are compared to values quoted

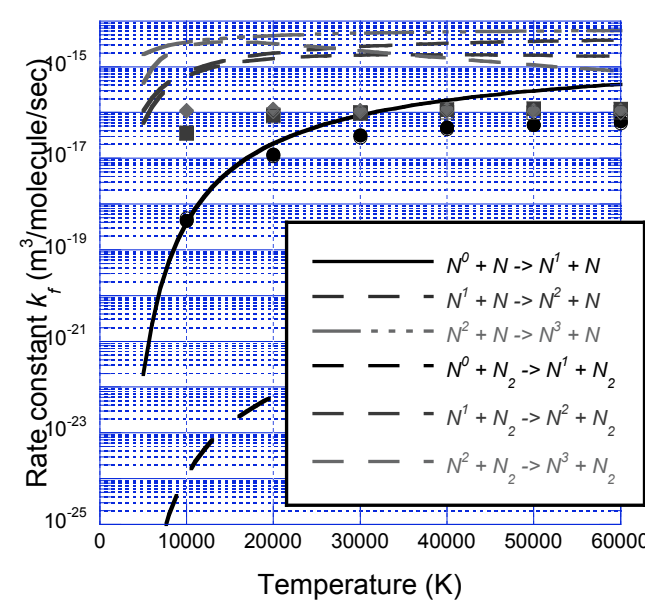

Figure 7. Heavy particle impact electronic energy level endothermic transition rates for $N_{2}$.

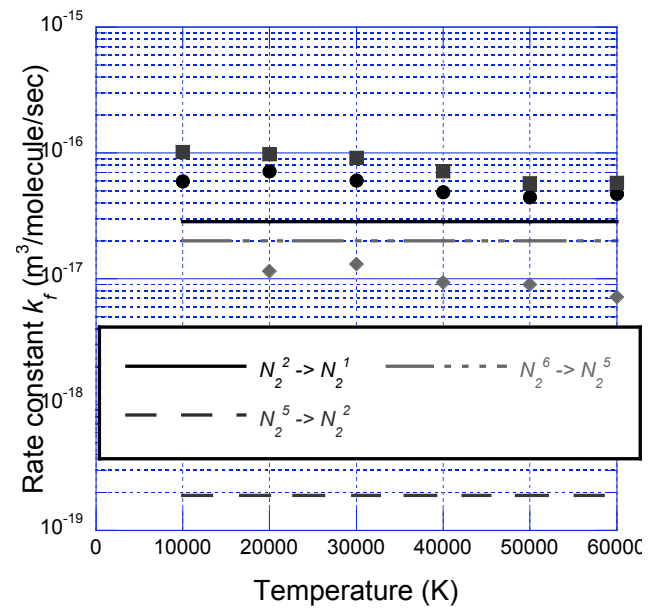

Figure 8. Heavy particle impact electronic energy level exothermic transition rates for $N_{2}$. 
by Levin ${ }^{22}$ in Fig. 9. Once again the quenching rates for electronic energy transitions compare reasonably well. Heavy particle impact ionization rates are not prevalent in the literature, so are not included in this analysis.

\section{Conclusion}

Kinetic theory based chemical reaction models recently proposed for the DSMC method have been extended to include electronic energy level transitions as well as ionization. This new set of models do not use measured macroscopic reaction/ transition rates to calibrate adjustable parameters. Instead, they make use of the principles of microscopic reversibility and molecular-level energy exchange to predict the probability that a chemical reaction or energy level transition occurs during a collision between two particles.

Procedures have been defined that are required in order to implement the current model for the distribution of electronic energy in the DSMC technique. These include defining methods to sample from an equilibrium (Boltzmann) distribution, to sample post-collision states from a combined

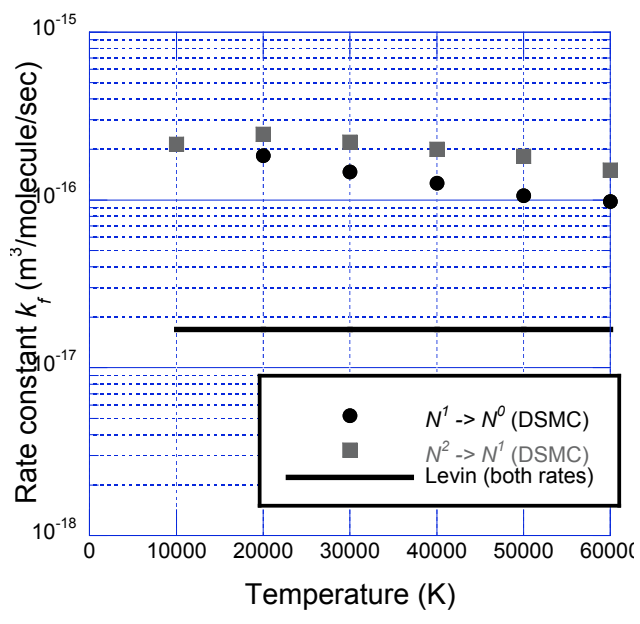

Figure 9. Heavy particle impact electronic energy level exothermic transition rates for $N$. distribution of the translational and electronic collision energies of the colliding particles, and to determine when a particle should transition electronic energy levels. The first two methods are straight forward and can be derived following methods for other energy modes. The last method is a novel implementation of the new chemical reaction models. These three methods have been shown to maintain equilibrium temperatures and reproduce the Boltzmann distribution of electronic energy levels after sufficient simulation time. Sampled electronic energy transition rates have been shown to compare well to values from literature for electron impact transitions and reasonably well for heavy particle impact transitions.

The new chemical reaction models have also been extended to include ionization reactions. Following the new dissociation model, where a molecule dissociates if the available collision energy exceeds the dissociation energy, a particle will loose an electron if the available collision energy exceeds the ionization energy. The sampled ionization rates have been shown to agree well with values from literature for electron impact reactions. However, heavy particle ionization data are scarce in literature and have been excluded from the current study.

\section{Appendix}

Table A.1. Listing of electronic energy level information of atomic nitrogen.

\begin{tabular}{|c|c|c|}
\hline Level $\boldsymbol{j}$ & $\boldsymbol{\varepsilon}_{\boldsymbol{e l}, \boldsymbol{j}} \mathbf{( J )}$ & $\boldsymbol{g}_{\boldsymbol{j}}$ \\
\hline $\mathbf{0}$ & 0.00000 & 1 \\
\hline $\mathbf{1}$ & $9.9726950 \mathrm{E}-19$ & 3 \\
\hline $\mathbf{2}$ & $1.1843030 \mathrm{E}-18$ & 6 \\
\hline $\mathbf{3}$ & $1.1880540 \mathrm{E}-18$ & 6 \\
\hline $\mathbf{4}$ & $1.3164670 \mathrm{E}-18$ & 3 \\
\hline $\mathbf{5}$ & $1.3538160 \mathrm{E}-18$ & 1 \\
\hline $\mathbf{6}$ & $1.3762700 \mathrm{E}-18$ & 2 \\
\hline $\mathbf{7}$ & $1.4482750 \mathrm{E}-18$ & 2 \\
\hline $\mathbf{8}$ & $1.5414820 \mathrm{E}-18$ & 5 \\
\hline $\mathbf{9}$ & $1.6924570 \mathrm{E}-18$ & 1 \\
\hline $\mathbf{1 0}$ & $1.7242390 \mathrm{E}-18$ & 6 \\
\hline $\mathbf{1 1}$ & $1.7706570 \mathrm{E}-18$ & 6 \\
\hline $\mathbf{1 2}$ & $1.8473930 \mathrm{E}-18$ & 10 \\
\hline $\mathbf{1 3}$ & $1.9388470 \mathrm{E}-18$ & 6 \\
\hline $\mathbf{1 4}$ & $2.0778240 \mathrm{E}-18$ & 6 \\
\hline
\end{tabular}


Table A.2. Listing of electronic energy level information of atomic nitrogen.

\begin{tabular}{|c|c|c|c|c|c|c|c|c|c|}
\hline Level $j$ & $\varepsilon_{e l, j}(\mathbf{J})$ & $g_{j}$ & $n_{j}$ & $l_{j}$ & $x_{j}$ & $S_{j}$ & $L_{j}$ & Config. & Term \\
\hline 0 & 0.00000 & 4 & 2 & 1 & 3 & $3 / 2$ & 0 & $2 s^{2} 2 p^{3}$ & ${ }^{4} \mathrm{~S}^{\circ}$ \\
\hline 1 & $3.8195294 \mathrm{E}-19$ & 10 & 2 & 1 & 3 & $1 / 2$ & 2 & $2 \mathrm{~s}^{2} 2 \mathrm{p}^{3}$ & ${ }^{2} \mathrm{D}^{\circ}$ \\
\hline 2 & $5.7287486 \mathrm{E}-19$ & 6 & 2 & 1 & 3 & $1 / 2$ & 1 & $2 s^{2} 2 p^{3}$ & ${ }^{2} \mathrm{Po}^{\circ}$ \\
\hline 3 & $1.6554172 \mathrm{E}-18$ & 12 & 3 & 0 & 1 & $3 / 2$ & 1 & $2 s^{2} 2 p^{2}\left({ }^{3} P\right) 3 s$ & ${ }^{4} \mathrm{P}$ \\
\hline 4 & $1.7121737 \mathrm{E}-18$ & 6 & 3 & 0 & 1 & $1 / 2$ & 1 & $2 s^{2} 2 p^{2}\left({ }^{3} P\right) 3 s$ & ${ }^{2} \mathrm{P}$ \\
\hline 5 & $1.7507040 \mathrm{E}-18$ & 12 & 2 & 1 & 4 & $3 / 2$ & 1 & $2 \mathrm{~s} 2 \mathrm{p}^{4}$ & ${ }^{4} \mathrm{P}$ \\
\hline 6 & $1.8589476 \mathrm{E}-18$ & 2 & 3 & 1 & 1 & $1 / 2$ & 0 & $2 s^{2} 2 p^{2}\left({ }^{3} P\right) 3 p$ & ${ }^{2} \mathrm{~S}^{\circ}$ \\
\hline 7 & $1.8839019 \mathrm{E}-18$ & 20 & 3 & 1 & 1 & $3 / 2$ & 2 & $2 s^{2} 2 p^{2}\left({ }^{3} P\right) 3 p$ & ${ }^{4} \mathrm{D}^{\circ}$ \\
\hline 8 & $1.8972523 \mathrm{E}-18$ & 12 & 3 & 1 & 1 & $3 / 2$ & 1 & $2 s^{2} 2 p^{2}\left({ }^{3} P\right) 3 p$ & ${ }^{4} \mathrm{Po}^{\circ}$ \\
\hline 9 & $1.9219038 \mathrm{E}-18$ & 4 & 3 & 1 & 1 & $3 / 2$ & 0 & $2 s^{2} 2 p^{2}\left({ }^{3} P\right) 3 p$ & ${ }^{4} \mathrm{~S}^{\circ}$ \\
\hline 10 & $1.9235458 \mathrm{E}-18$ & 10 & 3 & 1 & 1 & $1 / 2$ & 2 & $2 s^{2} 2 p^{2}\left({ }^{3} P\right) 3 p$ & ${ }^{2} \mathrm{D}^{\circ}$ \\
\hline 11 & $1.9426247 \mathrm{E}-18$ & 6 & 3 & 1 & 1 & $1 / 2$ & 1 & $2 s^{2} 2 p^{2}\left({ }^{3} P\right) 3 p$ & ${ }^{2} \mathrm{Po}^{\mathrm{o}}$ \\
\hline 12 & $1.9797645 \mathrm{E}-18$ & 10 & 3 & 0 & 1 & $1 / 2$ & 2 & $2 \mathrm{~s}^{2} 2 \mathrm{p}^{2}\left({ }^{1} \mathrm{D}\right) 3 \mathrm{~s}$ & ${ }^{2} \mathrm{D}$ \\
\hline 13 & $2.0598225 \mathrm{E}-18$ & 12 & 4 & 0 & 1 & $3 / 2$ & 1 & $2 s^{2} 2 p^{2}\left({ }^{3} P\right) 4 s$ & ${ }^{4} \mathrm{P}$ \\
\hline 14 & $2.0697973 \mathrm{E}-18$ & 6 & 4 & 0 & 1 & $1 / 2$ & 1 & $2 s^{2} 2 p^{2}\left({ }^{3} P\right) 4 s$ & ${ }^{2} \mathrm{P}$ \\
\hline 15 & $2.0783847 \mathrm{E}-18$ & 6 & 3 & 2 & 1 & $1 / 2$ & 1 & $2 s^{2} 2 p^{2}\left({ }^{3} P\right) 3 d$ & ${ }^{2} \mathrm{P}$ \\
\hline 16 & $2.0801974 \mathrm{E}-18$ & 28 & 3 & 2 & 1 & $3 / 2$ & 3 & $2 s^{2} 2 p^{2}\left({ }^{3} P\right) 3 d$ & ${ }^{4} \mathrm{~F}$ \\
\hline 17 & $2.0827249 \mathrm{E}-18$ & 12 & 3 & 2 & 1 & $3 / 2$ & 1 & $2 s^{2} 2 p^{2}\left({ }^{3} P\right) 3 d$ & ${ }^{4} \mathrm{P}$ \\
\hline 18 & $2.0828065 \mathrm{E}-18$ & 14 & 3 & 2 & 1 & $1 / 2$ & 3 & $2 s^{2} 2 p^{2}\left({ }^{3} P\right) 3 d$ & ${ }^{2} \mathrm{~F}$ \\
\hline 19 & $2.0859128 \mathrm{E}-18$ & 20 & 3 & 2 & 1 & $3 / 2$ & 2 & $2 s^{2} 2 p^{2}\left({ }^{3} P\right) 3 d$ & ${ }^{4} \mathrm{D}$ \\
\hline 20 & $2.0884332 \mathrm{E}-18$ & 10 & 3 & 2 & 1 & $1 / 2$ & 2 & $2 s^{2} 2 p^{2}\left({ }^{3} P\right) 3 d$ & ${ }^{2} \mathrm{D}$ \\
\hline 21 & $2.1151235 \mathrm{E}-18$ & 2 & 4 & 1 & 1 & $1 / 2$ & 0 & $2 s^{2} 2 p^{2}\left({ }^{3} P\right) 4 p$ & ${ }^{2} \mathrm{~S}^{0}$ \\
\hline 22 & $2.1219883 \mathrm{E}-18$ & 20 & 4 & 1 & 1 & $3 / 2$ & 2 & $2 s^{2} 2 p^{2}\left({ }^{3} P\right) 4 p$ & ${ }^{4} \mathrm{D}^{\circ}$ \\
\hline 23 & $2.1257740 \mathrm{E}-18$ & 12 & 4 & 1 & 1 & $3 / 2$ & 1 & $2 s^{2} 2 p^{2}\left({ }^{3} P\right) 4 p$ & ${ }^{4} \mathrm{Po}^{\circ}$ \\
\hline 24 & $2.1299658 \mathrm{E}-18$ & 10 & 4 & 1 & 1 & $1 / 2$ & 2 & $2 s^{2} 2 p^{2}\left({ }^{3} P\right) 4 p$ & ${ }^{2} \mathrm{D}^{\circ}$ \\
\hline 25 & $2.1343488 \mathrm{E}-18$ & 4 & 4 & 1 & 1 & $3 / 2$ & 0 & $2 s^{2} 2 p^{2}\left({ }^{3} P\right) 4 p$ & ${ }^{4} \mathrm{~S}^{\circ}$ \\
\hline 26 & $2.1377136 \mathrm{E}-18$ & 6 & 4 & 1 & 1 & $1 / 2$ & 1 & $2 s^{2} 2 p^{2}\left({ }^{3} P\right) 4 p$ & ${ }^{2} \mathrm{Po}^{\circ}$ \\
\hline 27 & $2.1827900 \mathrm{E}-18$ & 12 & 5 & 0 & 1 & $3 / 2$ & 1 & $2 s^{2} 2 p^{2}\left({ }^{3} P\right) 5 s$ & ${ }^{4} \mathrm{P}$ \\
\hline 28 & $2.1867017 \mathrm{E}-18$ & 6 & 5 & 0 & 1 & $1 / 2$ & 1 & $2 s^{2} 2 p^{2}\left({ }^{3} P\right) 5 s$ & ${ }^{2} \mathrm{P}$ \\
\hline 29 & $2.1896284 \mathrm{E}-18$ & 6 & 4 & 2 & 1 & $1 / 2$ & 1 & $2 s^{2} 2 p^{2}\left({ }^{3} P\right) 4 d$ & ${ }^{2} \mathrm{P}$ \\
\hline 30 & $2.1901014 \mathrm{E}-18$ & 28 & 4 & 2 & 1 & $3 / 2$ & 3 & $2 s^{2} 2 p^{2}\left({ }^{3} P\right) 4 d$ & ${ }^{4} \mathrm{~F}$ \\
\hline 31 & $2.1916419 \mathrm{E}-18$ & 12 & 4 & 2 & 1 & $3 / 2$ & 1 & $2 s^{2} 2 p^{2}\left({ }^{3} P\right) 4 d$ & ${ }^{4} \mathrm{P}$ \\
\hline 32 & $2.1916419 \mathrm{E}-18$ & 14 & 4 & 2 & 1 & $1 / 2$ & 3 & $2 s^{2} 2 p^{2}\left({ }^{3} P\right) 4 d$ & ${ }^{2} \mathrm{~F}$ \\
\hline 33 & $2.1920256 \mathrm{E}-18$ & 14 & 4 & 4 & 1 & $1 / 2$ & 3 & $2 s^{2} 2 p^{2}\left({ }^{3} P\right) 4 f D$ & $2[3]^{0}$ \\
\hline 34 & $2.1927445 \mathrm{E}-18$ & 14 & 4 & 4 & 1 & $1 / 2$ & 3 & $2 s^{2} 2 p^{2}\left({ }^{3} P\right) 4 f G$ & ${ }^{2}[3]^{0}$ \\
\hline 35 & $2.1930233 \mathrm{E}-18$ & 20 & 4 & 2 & 1 & $3 / 2$ & 2 & $2 s^{2} 2 p^{2}(3 P) 4 d$ & ${ }^{4} \mathrm{D}$ \\
\hline 36 & $2.1930782 \mathrm{E}-18$ & 18 & 4 & 4 & 1 & $1 / 2$ & 4 & $2 s^{2} 2 p^{2}\left({ }^{3} P\right) 4 f G$ & ${ }^{2}[4]^{0}$ \\
\hline 37 & $2.1931259 \mathrm{E}-18$ & 10 & 4 & 4 & 1 & $1 / 2$ & 2 & $2 s^{2} 2 p^{2}\left({ }^{3} P\right) 4 f D$ & ${ }^{2}[2]^{0}$ \\
\hline 38 & $2.1942236 \mathrm{E}-18$ & 6 & 4 & 4 & 1 & $1 / 2$ & 1 & $2 s^{2} 2 p^{2}\left({ }^{3} P\right) 4 f D$ & ${ }^{2}[1]^{0}$ \\
\hline 39 & $2.1942468 \mathrm{E}-18$ & 10 & 4 & 2 & 1 & $1 / 2$ & 2 & $2 \mathrm{~s}^{2} 2 \mathrm{p}^{2}\left({ }^{3} \mathrm{P}\right) 4 \mathrm{~d}$ & ${ }^{2} \mathrm{D}$ \\
\hline 40 & $2.1944891 \mathrm{E}-18$ & 22 & 4 & 4 & 1 & $1 / 2$ & 5 & $2 s^{2} 2 p^{2}\left({ }^{3} P\right) 4 f G$ & $2[5]^{0}$ \\
\hline 41 & $2.1947443 \mathrm{E}-18$ & 10 & 4 & 4 & 1 & $1 / 2$ & 2 & $2 s^{2} 2 p^{2}\left({ }^{3} P\right) 4 f F$ & ${ }^{2}[2]^{0}$ \\
\hline 42 & $2.1949910 \mathrm{E}-18$ & 14 & 4 & 4 & 1 & $1 / 2$ & 3 & $2 s^{2} 2 p^{2}\left({ }^{3} P\right) 4 f F$ & $2[3]^{0}$ \\
\hline 43 & $2.1950570 \mathrm{E}-18$ & 18 & 4 & 4 & 1 & $1 / 2$ & 4 & $2 \mathrm{~s}^{2} 2 \mathrm{p}^{2}\left({ }^{3} \mathrm{P}\right) 4 \mathrm{f} \mathrm{F}$ & ${ }^{2}[4]^{0}$ \\
\hline 44 & $2.1957241 \mathrm{E}-18$ & 10 & 3 & 1 & 1 & $1 / 2$ & 2 & $2 s^{2} 2 p^{2}\left({ }^{1} D\right) 3 p$ & ${ }^{2} \mathrm{D}^{\circ}$ \\
\hline 45 & $2.1992586 \mathrm{E}-18$ & 14 & 3 & 1 & 1 & $1 / 2$ & 3 & $2 s^{2} 2 p^{2}\left({ }^{1} D\right) 3 p$ & ${ }^{2} \mathrm{~F}^{\mathrm{o}}$ \\
\hline 46 & $2.2061644 \mathrm{E}-18$ & 2 & 5 & 1 & 1 & $1 / 2$ & 0 & $2 s^{2} 2 p^{2}\left({ }^{3} P\right) 5 p$ & ${ }^{2} \mathrm{~S}^{\circ}$ \\
\hline 47 & $2.2090956 \mathrm{E}-18$ & 6 & 5 & 1 & 1 & $1 / 2$ & 1 & $2 s^{2} 2 p^{2}\left({ }^{3} P\right) 5 p$ & ${ }^{2} \mathrm{Po}^{o}$ \\
\hline 48 & $2.2091778 \mathrm{E}-18$ & 20 & 5 & 1 & 1 & $3 / 2$ & 2 & $2 s^{2} 2 p^{2}\left({ }^{3} P\right) 5 p$ & ${ }^{4} \mathrm{D}^{\circ}$ \\
\hline 49 & $2.2092125 \mathrm{E}-18$ & 12 & 5 & 1 & 1 & $3 / 2$ & 1 & $2 s^{2} 2 p^{2}\left({ }^{3} P\right) 5 p$ & ${ }^{4} \mathrm{Po}^{\circ}$ \\
\hline 50 & $2.2149140 \mathrm{E}-18$ & 4 & 5 & 1 & 1 & $3 / 2$ & 0 & $2 s^{2} 2 p^{2}\left({ }^{3} P\right) 5 p$ & ${ }^{4} \mathrm{~S}^{\circ}$ \\
\hline 51 & $2.2225265 \mathrm{E}-18$ & 10 & 5 & 1 & 1 & $1 / 2$ & 2 & $2 s^{2} 2 p^{2}\left({ }^{3} P\right) 5 p$ & ${ }^{2} \mathrm{D}^{\circ}$ \\
\hline 52 & $2.2310009 \mathrm{E}-18$ & 6 & 3 & 1 & 1 & $1 / 2$ & 1 & $2 s^{2} 2 p^{2}\left({ }^{1} D\right) 3 p$ & ${ }^{2} \mathrm{Po}^{\circ}$ \\
\hline 53 & $2.2374954 \mathrm{E}-18$ & 12 & 6 & 0 & 1 & $3 / 2$ & 1 & $2 s^{2} 2 p^{2}\left({ }^{3} P\right) 6 s$ & ${ }^{4} \mathrm{P}$ \\
\hline
\end{tabular}

9

American Institute of Aeronautics and Astronautics 


\begin{tabular}{|c|c|c|c|c|c|c|c|c|c|}
\hline Level $j$ & $\varepsilon_{e l, j}(\mathbf{J})$ & $g_{j}$ & $n_{j}$ & $l_{j}$ & $x_{j}$ & $S_{j}$ & $L_{j}$ & Config. & Term \\
\hline 54 & $2.2391604 \mathrm{E}-18$ & 6 & 6 & 0 & 1 & $1 / 2$ & 1 & $2 \mathrm{~s}^{2} 2 \mathrm{p}^{2}\left({ }^{3} \mathrm{P}\right) 6 \mathrm{~s}$ & ${ }^{2} \mathrm{P}$ \\
\hline 55 & $2.2407743 \mathrm{E}-18$ & 6 & 5 & 2 & 1 & $1 / 2$ & 1 & $2 s^{2} 2 p^{2}\left({ }^{3} P\right) 5 d$ & ${ }^{2} \mathrm{P}$ \\
\hline 56 & $2.2408504 \mathrm{E}-18$ & 28 & 5 & 2 & 1 & $3 / 2$ & 3 & $2 s^{2} 2 p^{2}\left({ }^{3} P\right) 5 d$ & ${ }^{4} \mathrm{~F}$ \\
\hline 57 & $2.2412458 \mathrm{E}-18$ & 14 & 5 & 3 & 1 & $1 / 2$ & 3 & $2 \mathrm{~s}^{2} 2 \mathrm{p}^{2}\left({ }^{3} \mathrm{P}_{0}\right) 5 \mathrm{f}$ & ${ }^{2}[3]^{0}$ \\
\hline 58 & $2.2414379 \mathrm{E}-18$ & 12 & 5 & 2 & 1 & $3 / 2$ & 1 & $2 s^{2} 2 p^{2}\left({ }^{3} P\right) 5 d$ & ${ }^{4} \mathrm{P}$ \\
\hline 59 & $2.2418528 \mathrm{E}-18$ & 14 & 5 & 2 & 1 & $1 / 2$ & 3 & $2 s^{2} 2 p^{2}\left({ }^{3} P\right) 5 d$ & ${ }^{2} \mathrm{~F}$ \\
\hline 60 & $2.2420758 \mathrm{E}-18$ & 14 & 5 & 3 & 1 & $1 / 2$ & 3 & $2 s^{2} 2 p^{2}\left({ }^{3} P_{1}\right) 5 f$ & ${ }^{2}[3]^{0}$ \\
\hline 61 & $2.2422583 \mathrm{E}-18$ & 18 & 5 & 3 & 1 & $1 / 2$ & 4 & $2 \mathrm{~s}^{2} 2 \mathrm{p}^{2}\left({ }^{3} \mathrm{P}_{1}\right) 5 \mathrm{f}$ & ${ }^{2}[4]^{0}$ \\
\hline 62 & $2.2423070 \mathrm{E}-18$ & 10 & 5 & 3 & 1 & $1 / 2$ & 2 & $2 s^{2} 2 p^{2}\left({ }^{3} P_{1}\right) 5 f$ & ${ }^{2}[2]^{0}$ \\
\hline 63 & $2.2428934 \mathrm{E}-18$ & 20 & 5 & 2 & 1 & $3 / 2$ & 2 & $2 s^{2} 2 p^{2}\left({ }^{3} P\right) 5 d$ & ${ }^{4} \mathrm{D}$ \\
\hline 64 & $2.2434580 \mathrm{E}-18$ & 10 & 5 & 2 & 1 & $1 / 2$ & 2 & $2 s^{2} 2 p^{2}\left({ }^{3} P\right) 5 d$ & ${ }^{2} \mathrm{D}$ \\
\hline 65 & $2.2436230 \mathrm{E}-18$ & 6 & 5 & 3 & 1 & $1 / 2$ & 1 & $2 \mathrm{~s}^{2} 2 \mathrm{p}^{2}\left({ }^{3} \mathrm{P}_{2}\right) 5 \mathrm{f}$ & ${ }^{2}[1]^{0}$ \\
\hline 66 & $2.2437593 \mathrm{E}-18$ & 22 & 5 & 3 & 1 & $1 / 2$ & 4.5 & $2 \mathrm{~s}^{2} 2 \mathrm{p}^{2}\left({ }^{3} \mathrm{P}_{2}\right) 5 \mathrm{f}$ & ${ }^{2}[9 / 2]^{0}$ \\
\hline 67 & $2.2438446 \mathrm{E}-18$ & 10 & 5 & 3 & 1 & $1 / 2$ & 2 & $2 \mathrm{~s}^{2} 2 \mathrm{p}^{2}\left({ }^{3} \mathrm{P}_{2}\right) 5 \mathrm{f}$ & ${ }^{2}[2]^{0}$ \\
\hline 68 & $2.2439954 \mathrm{E}-18$ & 14 & 5 & 3 & 1 & $1 / 2$ & 3 & $2 \mathrm{~s}^{2} 2 \mathrm{p}^{2}\left({ }^{3} \mathrm{P}_{2}\right) 5 \mathrm{f}$ & ${ }^{2}[3]^{0}$ \\
\hline 69 & $2.2440307 \mathrm{E}-18$ & 18 & 5 & 3 & 1 & $1 / 2$ & 4 & $2 \mathrm{~s}^{2} 2 \mathrm{p}^{2}\left({ }^{3} \mathrm{P}_{2}\right) 5 \mathrm{f}$ & ${ }^{2}[4]^{0}$ \\
\hline 70 & $2.2515070 \mathrm{E}-18$ & 14 & 6 & 1 & 1 & $3 / 2$ & 2 & $2 s^{2} 2 p^{2}\left({ }^{3} \mathrm{P}\right) 6 \mathrm{p}$ & ${ }^{4} \mathrm{D}^{\circ}$ \\
\hline 71 & $2.2522583 \mathrm{E}-18$ & 10 & 6 & 1 & 1 & $3 / 2$ & 1 & $2 s^{2} 2 p^{2}\left({ }^{3} \mathrm{P}\right) 6 \mathrm{p}$ & ${ }^{4} \mathrm{Po}^{\circ}$ \\
\hline 72 & $2.2665626 \mathrm{E}-18$ & 12 & 7 & 0 & 1 & $3 / 2$ & 1 & $2 \mathrm{~s}^{2} 2 \mathrm{p}^{2}\left({ }^{3} \mathrm{P}\right) 7 \mathrm{~s}$ & ${ }^{4} \mathrm{P}$ \\
\hline 73 & $2.2679643 \mathrm{E}-18$ & 14 & 6 & 3 & 1 & $1 / 2$ & 3 & $2 s^{2} 2 p^{2}\left({ }^{3} P_{0}\right) 6 f$ & ${ }^{2}[3]^{0}$ \\
\hline 74 & $2.2680191 \mathrm{E}-18$ & 6 & 6 & 2 & 1 & $1 / 2$ & 1 & $2 s^{2} 2 p^{2}\left({ }^{3} P\right) 6 d$ & ${ }^{2} \mathrm{P}$ \\
\hline 75 & $2.2680197 \mathrm{E}-18$ & 6 & 7 & 0 & 1 & $1 / 2$ & 1 & $2 \mathrm{~s}^{2} 2 \mathrm{p}^{2}\left({ }^{3} \mathrm{P}\right) 7 \mathrm{~s}$ & ${ }^{2} \mathrm{P}$ \\
\hline 76 & $2.2682030 \mathrm{E}-18$ & 28 & 6 & 2 & 1 & $3 / 2$ & 3 & $2 s^{2} 2 p^{2}\left({ }^{3} P\right) 6 d$ & ${ }^{4} \mathrm{~F}$ \\
\hline 77 & $2.2686007 \mathrm{E}-18$ & 12 & 6 & 2 & 1 & $3 / 2$ & 1 & $2 s^{2} 2 p^{2}\left({ }^{3} P\right) 6 d$ & ${ }^{4} \mathrm{P}$ \\
\hline 78 & $2.2688525 \mathrm{E}-18$ & 14 & 6 & 3 & 1 & $1 / 2$ & 3 & $2 s^{2} 2 p^{2}\left({ }^{3} P_{1}\right) 6 f$ & ${ }^{2}[3]^{0}$ \\
\hline 79 & $2.2689594 \mathrm{E}-18$ & 18 & 6 & 3 & 1 & $1 / 2$ & 4 & $2 s^{2} 2 p^{2}\left({ }^{3} P_{1}\right) 6 f$ & ${ }^{2}[4]^{0}$ \\
\hline 80 & $2.2689951 \mathrm{E}-18$ & 10 & 6 & 3 & 1 & $1 / 2$ & 2 & $2 s^{2} 2 p^{2}\left({ }^{3} P_{1}\right) 6 f$ & ${ }^{2}[2]^{0}$ \\
\hline 81 & $2.2690781 \mathrm{E}-18$ & 14 & 6 & 2 & 1 & $1 / 2$ & 3 & $2 s^{2} 2 p^{2}\left({ }^{3} P\right) 6 d$ & ${ }^{2} \mathrm{~F}$ \\
\hline 82 & $2.2699818 \mathrm{E}-18$ & 20 & 6 & 2 & 1 & $3 / 2$ & 2 & $2 s^{2} 2 p^{2}\left({ }^{3} \mathrm{P}\right) 6 \mathrm{~d}$ & ${ }^{4} \mathrm{D}$ \\
\hline 83 & $2.2702472 \mathrm{E}-18$ & 10 & 6 & 2 & 1 & $1 / 2$ & 2 & $2 s^{2} 2 p^{2}\left({ }^{3} P\right) 6 d$ & ${ }^{2} \mathrm{D}$ \\
\hline 84 & $2.2704316 \mathrm{E}-18$ & 6 & 6 & 3 & 1 & $1 / 2$ & 1 & $2 s^{2} 2 p^{2}\left({ }^{3} P_{2}\right) 6 f$ & ${ }^{2}[1]^{0}$ \\
\hline 85 & $2.2705093 \mathrm{E}-18$ & 22 & 6 & 3 & 1 & $1 / 2$ & 5 & $2 s^{2} 2 p^{2}\left({ }^{3} P_{2}\right) 6 f$ & ${ }^{2}[5]^{0}$ \\
\hline 86 & $2.2705473 \mathrm{E}-18$ & 10 & 6 & 3 & 1 & $1 / 2$ & 2 & $2 s^{2} 2 p^{2}\left({ }^{3} P_{2}\right) 6 f$ & ${ }^{2}[2]^{0}$ \\
\hline 87 & $2.2706401 \mathrm{E}-18$ & 14 & 6 & 3 & 1 & $1 / 2$ & 3 & $2 s^{2} 2 p^{2}\left({ }^{3} P_{2}\right) 6 f$ & ${ }^{2}[3]^{0}$ \\
\hline 88 & $2.2706620 \mathrm{E}-18$ & 18 & 6 & 3 & 1 & $1 / 2$ & 4 & $2 s^{2} 2 p^{2}\left({ }^{3} P_{2}\right) 6 f$ & ${ }^{2}[4]^{0}$ \\
\hline 89 & $2.2753854 \mathrm{E}-18$ & 8 & 7 & 1 & 1 & $3 / 2$ & 2 & $2 s^{2} 2 p^{2}\left({ }^{3} P\right) 7 p$ & ${ }^{4} \mathrm{D}^{\circ}$ \\
\hline 90 & $2.2838695 \mathrm{E}-18$ & 12 & 8 & 0 & 1 & $3 / 2$ & 1 & $2 s^{2} 2 p^{2}\left({ }^{3} \mathrm{P}\right) 8 \mathrm{~s}$ & ${ }^{4} \mathrm{P}$ \\
\hline 91 & $2.2843827 \mathrm{E}-18$ & 6 & 7 & 2 & 1 & $1 / 2$ & 1 & $2 s^{2} 2 p^{2}\left({ }^{3} P\right) 7 d$ & ${ }^{2} \mathrm{P}$ \\
\hline 92 & $2.2846102 \mathrm{E}-18$ & 6 & 8 & 0 & 1 & $1 / 2$ & 1 & $2 \mathrm{~s}^{2} 2 \mathrm{p}^{2}\left({ }^{3} \mathrm{P}\right) 8 \mathrm{~s}$ & ${ }^{2} \mathrm{P}$ \\
\hline 93 & $2.2846711 \mathrm{E}-18$ & 28 & 7 & 2 & 1 & $3 / 2$ & 3 & $2 s^{2} 2 p^{2}\left({ }^{3} P\right) 7 d$ & ${ }^{4} \mathrm{~F}$ \\
\hline 94 & $2.2846925 \mathrm{E}-18$ & 10 & 7 & 2 & 1 & $3 / 2$ & 1 & $2 s^{2} 2 p^{2}\left({ }^{3} P\right) 7 d$ & ${ }^{4} \mathrm{P}$ \\
\hline 95 & $2.2854244 \mathrm{E}-18$ & 14 & 7 & 2 & 1 & $1 / 2$ & 3 & $2 s^{2} 2 p^{2}\left({ }^{3} P\right) 7 d$ & ${ }^{2} \mathrm{~F}$ \\
\hline 96 & $2.2862873 \mathrm{E}-18$ & 20 & 7 & 2 & 1 & $3 / 2$ & 2 & $2 s^{2} 2 p^{2}\left({ }^{3} P\right) 7 d$ & ${ }^{2} \mathrm{D}$ \\
\hline 97 & $2.2864728 \mathrm{E}-18$ & 10 & 7 & 2 & 1 & $1 / 2$ & 2 & $2 s^{2} 2 p^{2}\left({ }^{3} P\right) 7 d$ & ${ }^{2} \mathrm{D}$ \\
\hline 98 & $2.2939594 \mathrm{E}-18$ & 6 & 9 & 0 & 1 & $1 / 2$ & 1 & $2 \mathrm{~s}^{2} 2 \mathrm{p}^{2}\left({ }^{3} \mathrm{P}\right) 9 \mathrm{~s}$ & ${ }^{2} \mathrm{P}$ \\
\hline 99 & $2.2949085 \mathrm{E}-18$ & 12 & 9 & 0 & 1 & $3 / 2$ & 1 & $2 \mathrm{~s}^{2} 2 \mathrm{p}^{2}\left({ }^{3} \mathrm{P}\right) 9 \mathrm{~s}$ & ${ }^{4} \mathrm{P}$ \\
\hline 100 & $2.2950121 \mathrm{E}-18$ & 6 & 8 & 2 & 1 & $1 / 2$ & 1 & $2 s^{2} 2 p^{2}\left({ }^{3} P\right) 8 d$ & ${ }^{2} \mathrm{P}$ \\
\hline 101 & $2.2955104 \mathrm{E}-18$ & 24 & 8 & 2 & 1 & $3 / 2$ & 3 & $2 s^{2} 2 p^{2}\left({ }^{3} P\right) 8 d$ & ${ }^{4} \mathrm{~F}$ \\
\hline 102 & $2.2960411 \mathrm{E}-18$ & 14 & 8 & 2 & 1 & $1 / 2$ & 3 & $2 s^{2} 2 p^{2}\left({ }^{3} P\right) 8 d$ & ${ }^{2} \mathrm{~F}$ \\
\hline 103 & $2.2966880 \mathrm{E}-18$ & 12 & 8 & 2 & 1 & $3 / 2$ & 1 & $2 s^{2} 2 p^{2}\left({ }^{3} P\right) 8 d$ & ${ }^{4} \mathrm{P}$ \\
\hline 104 & $2.2967069 \mathrm{E}-18$ & 10 & 8 & 2 & 1 & $1 / 2$ & 2 & $2 s^{2} 2 p^{2}\left({ }^{3} P\right) 8 d$ & ${ }^{2} \mathrm{D}$ \\
\hline 105 & $2.2968674 \mathrm{E}-18$ & 20 & 8 & 2 & 1 & $3 / 2$ & 2 & $2 s^{2} 2 p^{2}\left({ }^{3} P\right) 8 d$ & ${ }^{4} \mathrm{D}$ \\
\hline 106 & $2.3011420 \mathrm{E}-18$ & 6 & 10 & 0 & 1 & $1 / 2$ & 1 & $2 s^{2} 2 p^{2}\left({ }^{3} P\right) 10 s$ & ${ }^{2} \mathrm{P}$ \\
\hline 107 & $2.3020713 \mathrm{E}-18$ & 6 & 9 & 2 & 1 & $1 / 2$ & 1 & $2 s^{2} 2 p^{2}\left({ }^{3} P\right) 9 d$ & ${ }^{2} \mathrm{P}$ \\
\hline 108 & $2.3020392 \mathrm{E}-18$ & 20 & 9 & 2 & 1 & $3 / 2$ & 2 & $2 s^{2} 2 p^{2}\left({ }^{3} P\right) 9 d$ & ${ }^{4} \mathrm{D}$ \\
\hline 109 & $2.3023276 \mathrm{E}-18$ & 14 & 9 & 2 & 1 & $1 / 2$ & 3 & $2 s^{2} 2 p^{2}\left({ }^{3} P\right) 9 d$ & ${ }^{2} \mathrm{~F}$ \\
\hline
\end{tabular}

10

American Institute of Aeronautics and Astronautics 


\begin{tabular}{|c|c|c|c|c|c|c|c|c|c|}
\hline Level $j$ & $\varepsilon_{e l, j}(\mathbf{J})$ & $g_{j}$ & $n_{j}$ & $l_{j}$ & $x_{j}$ & $S_{j}$ & $L_{j}$ & Config. & Term \\
\hline 110 & $2.3024486 \mathrm{E}-18$ & 12 & 10 & 0 & 1 & $3 / 2$ & 1 & $2 s^{2} 2 p^{2}\left({ }^{3} P\right) 10 s$ & ${ }^{4} \mathrm{P}$ \\
\hline 111 & $2.3039586 \mathrm{E}-18$ & 10 & 9 & 2 & 1 & $1 / 2$ & 2 & $2 s^{2} 2 p^{2}\left({ }^{3} P\right) 9 d$ & ${ }^{2} \mathrm{D}$ \\
\hline 112 & $2.3040740 \mathrm{E}-18$ & 12 & 9 & 2 & 1 & $3 / 2$ & 1 & $2 s^{2} 2 p^{2}\left({ }^{3} P\right) 9 d$ & ${ }^{4} \mathrm{P}$ \\
\hline 113 & $2.3063971 \mathrm{E}-18$ & 6 & 11 & 0 & 1 & $1 / 2$ & 1 & $2 s^{2} 2 p^{2}\left({ }^{3} P\right) 11 s$ & ${ }^{2} \mathrm{P}$ \\
\hline 114 & $2.3067336 \mathrm{E}-18$ & 12 & 11 & 0 & 1 & $3 / 2$ & 1 & $2 s^{2} 2 p^{2}\left({ }^{3} P\right) 11 s$ & ${ }^{4} \mathrm{P}$ \\
\hline 115 & $2.3073584 \mathrm{E}-18$ & 6 & 10 & 2 & 1 & $1 / 2$ & 1 & $2 s^{2} 2 p^{2}\left({ }^{3} P\right) 10 d$ & ${ }^{2} \mathrm{P}$ \\
\hline 116 & $2.3074386 \mathrm{E}-18$ & 14 & 10 & 2 & 1 & $1 / 2$ & 3 & $2 \mathrm{~s}^{2} 2 \mathrm{p}^{2}\left({ }^{3} \mathrm{P}\right) 10 \mathrm{~d}$ & ${ }^{2} \mathrm{~F}$ \\
\hline 117 & $2.3075347 \mathrm{E}-18$ & 20 & 10 & 2 & 1 & $3 / 2$ & 2 & $2 \mathrm{~s}^{2} 2 \mathrm{p}^{2}\left({ }^{3} \mathrm{P}\right) 10 \mathrm{~d}$ & ${ }^{4} \mathrm{D}$ \\
\hline 118 & $2.3090407 \mathrm{E}-18$ & 10 & 10 & 2 & 1 & $1 / 2$ & 2 & $2 s^{2} 2 p^{2}\left({ }^{3} P\right) 10 d$ & ${ }^{2} \mathrm{D}$ \\
\hline 119 & $2.3094253 \mathrm{E}-18$ & 12 & 10 & 2 & 1 & $3 / 2$ & 1 & $2 s^{2} 2 p^{2}\left({ }^{3} P\right) 10 d$ & ${ }^{4} \mathrm{P}$ \\
\hline 120 & $2.3098102 \mathrm{E}-18$ & 2 & 3 & 0 & 1 & $1 / 2$ & 0 & $2 s^{2} 2 p^{2}\left({ }^{1} S\right) 3 s$ & ${ }^{2} \mathrm{~S}$ \\
\hline 121 & $2.3103385 \mathrm{E}-18$ & 6 & 12 & 0 & 1 & $1 / 2$ & 1 & $2 s^{2} 2 p^{2}\left({ }^{3} P\right) 12 s$ & ${ }^{2} \mathrm{P}$ \\
\hline 122 & $2.3104667 \mathrm{E}-18$ & 12 & 12 & 0 & 1 & $3 / 2$ & 1 & $2 s^{2} 2 p^{2}\left({ }^{3} P\right) 12 s$ & ${ }^{4} \mathrm{P}$ \\
\hline 123 & $2.3112517 \mathrm{E}-18$ & 6 & 11 & 2 & 1 & $1 / 2$ & 1 & $2 s^{2} 2 p^{2}\left({ }^{3} P\right) 11 d$ & ${ }^{2} \mathrm{P}$ \\
\hline 124 & $2.3114120 \mathrm{E}-18$ & 14 & 11 & 2 & 1 & $1 / 2$ & 3 & $2 s^{2} 2 p^{2}\left({ }^{3} P\right) 11 d$ & ${ }^{2} \mathrm{~F}$ \\
\hline 125 & $2.3115722 \mathrm{E}-18$ & 20 & 11 & 2 & 1 & $3 / 2$ & 2 & $2 s^{2} 2 p^{2}\left({ }^{3} P\right) 11 d$ & ${ }^{4} \mathrm{D}$ \\
\hline 126 & $2.3129340 \mathrm{E}-18$ & 10 & 11 & 2 & 1 & $1 / 2$ & 2 & $2 s^{2} 2 p^{2}\left({ }^{3} P\right) 11 d$ & ${ }^{2} \mathrm{D}$ \\
\hline 127 & $2.3130302 \mathrm{E}-18$ & 12 & 11 & 2 & 1 & $3 / 2$ & 1 & $2 s^{2} 2 p^{2}\left({ }^{3} P\right) 11 d$ & ${ }^{4} \mathrm{P}$ \\
\hline 128 & $2.3135589 \mathrm{E}-18$ & 6 & 13 & 0 & 1 & $1 / 2$ & 1 & $2 s^{2} 2 p^{2}\left({ }^{3} P\right) 13 s$ & ${ }^{2} \mathrm{P}$ \\
\hline 129 & $2.3142478 \mathrm{E}-18$ & 6 & 12 & 2 & 1 & $1 / 2$ & 1 & $2 s^{2} 2 p^{2}\left({ }^{3} P\right) 12 d$ & ${ }^{2} \mathrm{P}$ \\
\hline 130 & $2.3158179 \mathrm{E}-18$ & 12 & 12 & 2 & 1 & $3 / 2$ & 1 & $2 s^{2} 2 p^{2}\left({ }^{3} P\right) 12 d$ & ${ }^{4} \mathrm{P}$ \\
\hline 131 & $2.3166991 \mathrm{E}-18$ & 10 & 12 & 2 & 1 & $1 / 2$ & 2 & $2 s^{2} 2 p^{2}\left({ }^{3} P\right) 12 d$ & ${ }^{2} \mathrm{D}$ \\
\hline
\end{tabular}

\section{Bibliography}

1. Carlson, A. B., and Hassan, H. A. "Radiation Modeling with Direct Simulation Monte Carlo," Journal of Thermophysics and Heat Transfer Vol. 6, 1992, pp. 631-636.

2. $\quad$ Berghausen, A. K., Taylor, J. C., and Hassan, H. A. "Direct Simulation of Shock Front Radiation in Air," Journal of Thermophysics and Heat Transfer Vol. 10, No. 3, 1996, p. 6.

3. Kossi, K. K., and Boyd, I. D. "Detailed computation of Ultraviolet Spectra in Rarefied Hypersonic Flow," Journal of Thermophysics and Heat Transfer Vol. 35, 1998, pp. 653-659.

4. $\quad$ Park, C. Nonequilibrium Hypersonic Aerothermodynamics. New York: Wiley, 1990.

5. Bird, G. A. "A Comparison of Collision Energy-based and Temperature-based Procedures in DSMC," Rarefied Gas Dynamics, 26th Symposium. Vol. 1084, American Institue of Physics, Kyoto, Japan, 2009, pp. 245-250.

6. Gallis, M. A., Bond, R. B., and Torczynski, J. R. "A kinetic-theory approach for computing chemicalreaction rates in upper-atmosphere hypersonic flows," The Journal of Chemical Physics Vol. 131, No. 12, 2009, p. 124311.

7. Borgnakke, C., and Larsen, P. S. "Statistical collision model for Monte Carlo simulation of polyatomic gas mixtures," Journal of Computational Physics Vol. 18, 1975.

8. Bergemann, F., and Boyd, I. D. "New Discrete Vibrational Energy Model for Direct Simulation Monte Carlo Method," Progess in Astronautics and Aeronautics Vol. 160, 1994, pp. 174-183.

9. Boyd, I. D. "Relaxation of Discrete Rotational Energy Distributions Using a Monte Carlo Method," Physics of Fluids A Vol. 5, No. 9, 1993, pp. 2278-2286.

10. Bird, G. A. "Monte Carlo simulation in an engineering context," Progess in Astronautics and Aeronautics Vol. 74, No. 1, 1981.

11. Koura, K., and Matsumoto, H. "Variable soft sphere molecular model for inverse-power-law or LennardJones potential," Physics of Fluids A Vol. 3, 1991. 
12. Chernyi, G. G., and Losev, S. A. "Development of Thermal Protection Systems for Interplanetary Flight," ISTC Report. Research Institute of Mechanics, Moscow, 1999.

13. Frost, R. M., Awakowicz, P., Summers, H. P., and Badnell, N. R. "Calculated cross sections and measured rate coefficients for electron-impact excitation of neutral and singly ionized nitrogen," Journal of Applied Physics Vol. 84, No. 6, 1998, pp. 2989-3003.

14. Losev, S. A., Makarov, V. N., Pogosbekyan, M. J., Shatalov, O. P., and Nikol'sky, V. S. "Thermochemical Nonequilibrium Kinetic Models in Strong Shock Waves on Air." AIAA 1994-1990, 1994.

15. Ozawa, T., Zhong, J., and Levin, D. A. "Development of kinetic-based energy exchange models for noncontinuum, ionized hypersonic flows," Physics of Fluids Vol. 20, 2008.

16. Bird, G. A. "Nonequilibrium Radiation During Re-Entry at 10 km/s." AIAA 1987-1543, 1987.

17. Hash, D., Olejniczak, J., Wright, M., Prabhu, D., Pulsonetti, M., Hollis, B., Gnoffo, P., Barnhardt, M., Nompelis, I., and Candler, G. "FIRE II Calculations for Hypersonic Nonequilibrium Aerothermodynamics Code Verification: DPLR, LAURA and US3D." AIAA 2007-605, 2007.

18. Boyd, I. D. "Modeling of Plasma Formation in Rarefied Hypersonic Entry Flows." AIAA 2007-206, 2007.

19. Gupta, R. N. "Navier-Stokes and Viscous Shock-Layer Solutions for Radiating Hypersonic Flows." AIAA 1987-1576, 1987.

20. Park, C. "Rate Parameters for Electronic Excitation of Diatomic Molecules II. Heavy Particle-Impact Processes." AIAA 2008-1446, 2008.

21. Guerra, V., and Loureiro, J. "Electron and heavy particle kinetics in a low-pressure nitrogen glow discharge," Plasma Sources Science \& Technology Vol. 6, 1997, pp. 361-372.

22. Levin, D. A. "Modeling of VUV radiation at high altitudes." AIAA 1996-1899, 1996. 